\title{
Risk Aversion, Indivisible Timing Options and Gambling ${ }^{\dagger}$
}

\author{
Vicky Henderson \\ University of Oxford \\ David Hobson ${ }^{\S}$ \\ University of Warwick
}

May 20, 2011

\begin{abstract}
In this paper we model the behavior of a risk averse agent who seeks to maximize expected utility and who has an indivisible asset and a timing option over when to sell this asset. Our main contribution is to show that, contrary to intuition, optimal behavior for such a risk averse agent can include risk increasing gambles. For example, a manager with a choice over when to disinvest from a project, a private homeowner with a property to sell, or an employee with a grant of American-style stock options may be better off taking positions in other assets with zero Sharpe ratio which are uncorrelated with the underlying project, house or stock price risk. The results have wider implications for the modeling and interpretation of portfolio optimization problems involving American style timing decisions.
\end{abstract}

Keywords and Phrases: Timing option, American options, Optimal stopping, dynamic programming, Gambling, Incomplete markets, Portfolio choice, Sequential lotteries.

JEL Classification Numbers: D52, D81, G11, G12, G31

\footnotetext{
${ }^{\dagger}$ The authors would like to thank Hans Föllmer, Anthony Neuberger, José Scheinkman, Hyun Song Shin, Richard Stanton and Dick Stapleton for comments. The research was started whilst the first author was at Princeton University and partially supported by NSF grant DMI 0447990.

${ }^{\ddagger}$ Oxford-Man Institute, University of Oxford, Eagle House, Walton Well Rd, Oxford. OX2 6ED, UK. Email: vicky.henderson@oxford-man.ox.ac.uk

${ }^{\S}$ University of Warwick, Coventry, CV4 7AL, UK. Email: d.hobson@warwick.ac.uk
} 
It is a general principle that it is never optimal for an agent with increasing, strictly concave von Neumann-Morgenstern preferences to enter into a fair gamble, in the sense that to do so reduces her expected utility. In this paper we describe a converse to this principle. We consider the problem facing a risk averse agent who owns the rights to an indivisible asset. For example the agent may be a private homeowner, or the owner-manager of a company with an asset a parcel of land, a factory or a patent - to sell. The agent wishes to choose the optimal time to sell this asset in order to maximize her expected utility of wealth. Our main result is that, contrary to naive intuition, the agent can improve her expected utility by gambling — in other words the timing option may make the agent locally risk seeking. In blunt terms, our analysis suggests that before selling the asset an agent may benefit from a trip to a casino.

In our model a risk averse agent chooses when to sell an indivisible asset. If the asset is depreciating on average then the agent will always choose to sell immediately, but if it is appreciating, then depending on the relative risk and return, the agent may wish to delay the sale. Furthermore, there may be return distributions for which the agent's decision about whether to sell now or later depends on her current wealth. (If she has decreasing absolute risk aversion then as her wealth increases she becomes more tolerant to risk. Postponing the sale of the asset is akin to accepting the risk of price movements. The agent is more willing to accept this risk if she is richer.) The key point is that at a point of indifference the agent's indirect utility (formed by taking the maximum over the alternative strategies of selling and waiting) is locally convex in her wealth level. For this reason it makes sense for the agent to undertake a gamble, provided that she can defer the decision about when to sell the asset until the result of this gamble is known.

This result has many potential applications. A student, who, if only she were richer, would return to college for a graduate degree which may increase her net human capital, would be justified in buying lottery tickets. An individual inheriting a property, and indifferent between selling that property in today's depressed housing market or bearing the risk of price moves, might take a highly-geared short-term stock market position, and bear the house price risk if this investment produces gains. A political candidate unsure whether to continue her campaign for office might announce a controversial opinion in the hope that this might increase her public standing, and on the understanding that she would withdraw her candidacy if this opinion proved unpopular.

We will focus much of our discussion on applications to real options and executive compensation since, as we will explain, their features naturally fall into our framework. A crucial feature of our problem is a timing decision. Timing problems arise naturally in the context of real options (see Brennan and Schwartz (1985), McDonald and Siegel (1986), and Dixit and Pindyck (1994)) and executive stock options (see for example, Hall and Murphy (2000)). ${ }^{1}$ Indeed, these problems have their origins in the work of McKean (1965), Samuelson (1965) and Merton (1973) on American options. The sale or exercise decisions in these settings are typically indivisible (typically it is not easy to sell half of a house or factory). ${ }^{2}$ Real options problems are typically incomplete because of the non-tradability of the asset underlying the option (see for example, Smith and Nau (1995), Smith and McCardle (1998)). Executives with a grant of options or restricted stock face hedging restrictions imposed by law.

In the recent literature, the American exercise (or sale) timing problem is considered in tan-

\footnotetext{
${ }^{1}$ Some more recent developments in this literature include the effect of macroeconomic conditions (Guo et al (2005)), learning (Décamps and Mariotti(2004)), incomplete information (Décamps, Mariotti, and Villeneuve (2005) and the impact of a one-time investment on the exit decision (Kwon (2010)).

${ }^{2}$ Whilst executive stock options can be exercised inter-temporally, most models treat the options as a block, and, in practice, most are exercised in large tranches.
} 
dem with the agent's wider portfolio choice problem, giving mixed control/stopping problems. An example of this in a complete market setting is Karatzas and Wang (2000). However we are concerned with incomplete markets, and some examples in the real options literature include Hugonnier and Morellec (2007), Miao and Wang (2007), Henderson (2007) and Evans, Henderson and Hobson (2008). Joint control/stopping models in the context of executive stock options include those of Detemple and Sundaresan (1999), Kahl, Liu and Longstaff (2003), Ingersoll (2006), Leung and Sircar (2009) and Carpenter, Stanton and Wallace (2010). Optimal stopping problems are solved via a free boundary problem and the associated Hamilton-Jacobi-Bellman equation. A candidate solution is found and verified using the value-matching and smooth-pasting conditions. However, it is somewhat rare for solutions to mixed control/stopping problems to be available analytically, and many of the papers mentioned above solve the free boundary problem using numerical methods.

We turn now to think about the optimal control component of the models. The agent has access to other traded assets (such as the stock market) and may wish to include these in her optimal position. If the traded assets are correlated with the unhedgeble asset, then the agent would want to hold them for hedging purposes. ${ }^{3}$ Such assets enable the agent to reduce the risk exposure inherent in their real option or executive stock option position. Similarly, if the traded asset has a positive Sharpe ratio, then this provides an investment motive to hold the asset. In these strands of the literature, it is not surprising that a risk averse manager will find the access to trading opportunities desirable, firstly, as they enable her to hedge, and secondly, because they are beneficial due to their positive Sharpe ratio.

Our continuous-time, sale-timing model makes a contrasting and important observation to this literature. We show that even in the absence of these ingredients (positive expected return and correlation), a risk averse agent with a timing choice can benefit from the ability to trade in another asset - such trading is effectively an independent, zero expected return investment or fair gamble. These gambles are risk-increasing, rather than the risk-decreasing opportunities offered by a correlated asset. From the perspective of portfolio choice modeling, our observation demonstrates that seemingly extraneous assets can form part of an agent's optimal portfolio. Put differently, in such models with a decision over timing choice (when to exercise or sell) together with the ability to trade other outside assets, the special case where the trading opportunities have zero correlation and zero Sharpe ratio does not recover the simpler model without the trading opportunities. This is important because such simpler models are often used as a benchmark case, particularly when the full model can only be solved numerically. Our results also enable us to reinterpret results of Carpenter, Stanton and Wallace (2010). In a continuous time setting, they model an agent with CRRA who is compensated with (indivisible) American call options with finite maturity. The agent can dynamically invest in the financial market which is correlated with the company stock. Since there are three state variables (time, stock price and wealth) this framework leads to a free-boundary problem which is solved numerically. For numerical stability, they put bounds on the position held in the traded/market asset. The desire to hold the traded asset arises from features present in their model (positive Sharpe ratio, correlation and convex payoff); however, in light of our model, the desire to hold very large quantities of the traded asset can be seen to be due to the indivisibility and timing embedded in their problem. This desire to hold large (even infinite) positions is reflected in the difficulty of obtaining a numerical solution.

Our result offers a rational explanation for the occurrence of gambling by risk averse agents.

\footnotetext{
${ }^{3}$ Hedging motives are important also in portfolio choice problems in incomplete markets which do not involve timing problems, such as models with stochastic labor income, see Campbell and Viceira (2002). Again, in this context, access to financial markets enables an agent to offset some of the unhedgeable labor income risk.
} 
Other justifications for gambling exist in the literature, both rational and irrational. Many occurrences of gambling in the literature can be traced to the agent having a non-concave objective function. One example is risk-neutral equity-holders of a levered firm preferring asset price volatility (the "gambling for resurrection" of Jensen and Meckling (1976) or in continuous time, see Leland (1998)). Another example is the potential risk-taking incentives induced by stock options - this may arise from a manager influencing firm value through project choice, or fund managers who control the risky investments of their fund. In each of these contexts, gambling refers to volatility or uncertainty in the asset itself (be that the stock underlying the options, firm value, or fund value). In contrast, gambling in our context takes place in other outside assets.

Much of this literature employs static models, for instance, Ross (2004) analyzes the question of risk taking incentives and decomposes these into a convexity, translation and magnification effect. Carpenter (2000) studies similar issues in a continuous time model where a fund manager is compensated with an explicit convex payoff and can dynamically adjust fund volatility. However, there is no timing effect present as the compensation is received at a fixed horizon date. A further interesting paper of Basak, Pavlova and Shapiro (2007) investigates the implications of fund manager compensation involving an implicit non-concave payoff which arises from fund flows, and find this may encourage (price) risk-taking behavior. Relative to this literature, we show gambling (in outside assets) can be optimal without the presence of convex payoffs, but when the agent faces an indivisible timing problem. Given many situations in this literature involve payoffs with timing features, this is clearly of economic importance. The distinction between price-risk taking and gambling in outside assets (or convexity in price versus wealth) is important - firstly, gambling in outside assets is not universally attractive but depends on preferences. For example, gambling in outside assets will not occur under CARA but the pricerisk taking of Ross (2004) and Carpenter (2000) will. Secondly, it is easier to envisage agents being able to gamble using outside assets (financial markets, or even casinos) but more difficult to imagine that many agents (except top executives) are able to influence the price of the asset under consideration. These differences enable our model to capture economic situations which are not dealt with in the executive/fund manager compensation literature. In particular, our model involves a timing choice, but not a explicit (or implicit) convex payoff. ${ }^{4}$

Irrational justifications for gambling include the agent being overconfident about small probabilities (buying lottery tickets for example), receiving utility from the act of gambling itself and the existence of reference levels — "A person who has not made peace with his losses is likely to accept gambles which would be unacceptable to him otherwise" (Kahneman and Tversky (1979)). In a behavioral model with reference levels, Barberis and Xiong (2011) find that discounting of relative gains and losses (and the delaying of realizing losses) encourages the investor to prefer high price volatility. There is a similar interaction between discounting and logarithmic utility in Karatzas and Wang (2000). Again this desire for increased volatility refers to the indivisible asset, and does not equate to the gambling in outside wealth that we propose. The explanation we give is distinct from these in that we retain a fully rational framework in which the agent maximizes expected utility for a concave utility function, and for the correct probabilities.

In this paper we will consider two versions of the indivisible asset sale problem, one in a single-period discrete time model, and one in continuous time. The discrete formulation enables

\footnotetext{
${ }^{4}$ Whilst we emphasize the results for the asset sale situation whereby the agent receives the payoff $Y$ at a future time of her choice, we can adapt the results to show similar qualitative findings for other payoffs $h(Y)$. Whilst this enables our results to generalize to cover option payoffs, it also introduces an explicit price convexity ( $h$ is convex) which should be distinguished from our finding of convexity in wealth.
} 
us to demonstrate clearly the phenomena, whilst the continuous time framework is closer to the literature on optimal exercise or sale problems. It is also interesting to consider the similarities and differences between the two set-ups. We will show that, apart from a few special cases, the phenomenon whereby the option to choose timing of the sale induces gambling is always present in the discrete time model, but only sometimes present in the continuous model. In both set-ups, we show there are substantial welfare gains to be made by accessing gambles.

The essential features which drive the conclusion that it is optimal to gamble are that the agent has a timing option, that the asset for sale is indivisible, and that frictions ensure the market is incomplete, so that the decision to delay sale exposes the agent to unhedgeable risk. Between them these features lead to the counter-intuitive conclusion that it is optimal for a risk averse agent to gamble. This phenomenon is seen in a wide range of situations, but cannot occur if the market is complete, if the expected sale price of the asset is not increasing, or if the asset can be partially sold.

\section{Risk Aversion and Gambling in a One-period Model}

Consider a risk averse agent with initial wealth $x$ and utility function $u$, and suppose that the agent has an indivisible asset to sell. The asset can either be sold today for a certain amount ${ }^{5}$ $y$, or sold one time unit later for the random amount $Y$. In the absence of any other investment opportunities or risks the maximum expected utility of the agent is

$$
V_{0}(x)=\max \{u(x+y), \mathbb{E}[u(x+Y)]\} .
$$

For fixed $x, u$ and $y$ we say that $Y$ is advantageous if $\mathbb{E}[u(x+Y)]>u(x+y)$.

Now fix $u, y$ and $Y$ and allow wealth $x$ to vary. There are three possible scenarios: either $u(x+y)>\mathbb{E}[u(x+Y)]$ for all $x$, it is never optimal to delay sale and we say that $Y$ is never advantageous; or $u(x+y)<\mathbb{E}[u(x+Y)]$ for all $x$, it is always optimal to delay the sale, and we say $Y$ is always advantageous; or it is possible that as $x$ varies we can have both $u(x+y)>\mathbb{E}[u(x+Y)]$ and $u(x+y)<\mathbb{E}[u(x+Y)]$. In a sense the first two scenarios are degenerate. We are primarily interested in the consequences which follow in the last scenario, which we characterize by saying that $Y$ is sometimes advantageous.

Suppose that we are in the third scenario and that the agent has the opportunity to enter into an independent fair gamble $G$. Suppose further that the results of the gamble are known prior to the time at which the agent decides whether to sell the asset or not. It follows that the maximum expected utility is

$$
V_{G}(x)=\mathbb{E}\left[V_{0}(x+G)\right]=\mathbb{E}[\max \{u(x+y+G), \mathbb{E}[u(x+Y+G) \mid G]\}]
$$

The unexpected result is that in many cases $V_{0}(x)<V_{G}(x)$, even though the agent is risk averse, and the gamble is fair.

\subsection{DARA utilities}

Suppose the utility function has (strictly) decreasing absolute risk aversion ${ }^{6}$, and fix the constant $y$ and the random variable $Y$. We begin by showing that if an agent with DARA utility and initial

\footnotetext{
${ }^{5}$ For the purposes of this section it is possible to absorb the current value of the asset into the wealth of the agent, and thence without loss of generality to take $y=0$. However, we choose to keep $y$ in the notation to facilitate comparison with the continuous time case.

${ }^{6}$ Set $r(x)=-u^{\prime \prime}(x) / u^{\prime}(x)$. Then $r$ is the Arrow-Pratt coefficient of absolute risk aversion (Pratt (1964), Arrow (1971)).
} 
wealth $x$ would choose to wait before selling, then the same agent would also choose to wait if she were richer. ${ }^{7}$

Lemma 1 Suppose $u$ exhibits DARA, and suppose $x$ is such that $\mathbb{E}[u(x+Y)]>u(x+y)$. Then for all $z>x, \mathbb{E}[u(z+Y)]>u(z+y)$.

Proof: By hypothesis, for $v>y, u^{\prime \prime}(x+v)>\left(u^{\prime \prime}(x+y) / u^{\prime}(x+y)\right) u^{\prime}(x+v)$, so that integrating from $v=y$ to $v=z$,

$$
u^{\prime}(x+z)-u^{\prime}(x+y)>\frac{u^{\prime \prime}(x+y)}{u^{\prime}(x+y)}(u(x+z)-u(x+y)) .
$$

For $v<y$ the inequality is reversed, but when we now integrate from $z$ to $x$ we again find that (3) holds. (By interchanging the roles of $y$ and $z$ it can be shown that (3) is equivalent to the statement that $u$ is DARA, but we will only use the forward implication.)

Define $Q(x)=\mathbb{E}[u(x+Y)]-u(x+y)$ so that $Y$ is advantageous at $x$ if $Q(x)>0$. Then

$$
\begin{aligned}
Q^{\prime}(x) & =\mathbb{E}\left[u^{\prime}(x+Y)\right]-u^{\prime}(x+y) \\
& >\frac{u^{\prime \prime}(x+y)}{u^{\prime}(x+y)}(\mathbb{E}[u(x+Y)]-u(x+y)) \\
& =\frac{u^{\prime \prime}(x+y)}{u^{\prime}(x+y)} Q(x) .
\end{aligned}
$$

It follows that if $Q\left(x^{*}\right)=0$ then $Q^{\prime}\left(x^{*}\right)>0$, so that if there exists $x$ for which $Q(x)>0$ then $Q(z)>0$ for all $z>x$.

Corollary 2 Let $I=I(u, y, Y)$ be the set of wealths $x$ such that $Y$ is advantageous. Then $I$ is an interval which is unbounded on the right.

Theorem 3 Suppose $u$ is DARA and that $Y$ is sometimes advantageous. Then there are initial wealths $x$ at which it is optimal to gamble.

Proof: By assumption, there are wealths for which $Q(x)$ takes either sign, and then since $Q$ is continuous (at least when finite), by the intermediate value theorem there exists $x^{*}$ for which $Q\left(x^{*}\right)=0$, and then $Q^{\prime}\left(x^{*}\right)>0$.

Let $Z$ be the symmetric random variable on \pm 1 , and let $\epsilon$ be a small parameter. Suppose the agent has initial wealth $x^{*}$ and consider the strategy whereby the agent enters the fair gamble with return $\epsilon Z$, and then contingent on the outcome of the gamble, sells immediately if $Z=-1$ and waits if $Z=+1$.

Then $V_{\epsilon Z}\left(x^{*}\right)=\mathbb{E}\left[u\left(x^{*}+(y-\epsilon) I_{\{Z=-1\}}+(Y+\epsilon) I_{\{Z=1\}}\right)\right]=\left(u\left(x^{*}-\epsilon+y\right)+\mathbb{E}\left[u\left(x^{*}+\epsilon+Y\right)\right]\right) / 2$ and

$$
\left.\frac{d V_{\epsilon Z}}{d \epsilon}\right|_{\epsilon=0}=\frac{1}{2}\left(-u^{\prime}\left(x^{*}+y\right)+\mathbb{E}\left[u^{\prime}\left(x^{*}+Y\right)\right]\right)=\frac{1}{2} Q^{\prime}\left(x^{*}\right)>0
$$

In particular, for a sufficiently small $\epsilon$ the agent can improve her expected utility by gambling.

\footnotetext{
${ }^{7}$ Lemma 1 and Corollary 2 are special cases of Theorem 2 of Pratt (1964) and the main result in Dybvig and Lippman (1983) but we provide (new) proofs, partly for completeness, and partly to introduce notation for other results.
} 


\subsection{Non-DARA utilities}

The class of DARA utilities is a large and realistic class of utility functions, which includes the constant relative risk aversion (CRRA) utilities. However, it is interesting to consider the extent to which the results of Section 1.1 generalize to other utilities.

Firstly suppose that $u$ has constant absolute risk aversion, so that up to scaling $u(x)=$ $-e^{-\eta x} / \eta$. Then, for $G$ non-zero, the independence of $Y$ and $G$ gives

$$
\begin{aligned}
\mathbb{E}[\max \{u(x+y+G), \mathbb{E}[u(x+Y+G) \mid G]\}] & =-\frac{1}{\eta} e^{-\eta(x+y)} \mathbb{E}\left[e^{-\eta G}\right] \max \left\{1, \mathbb{E}\left[e^{-\eta(Y-y)}\right]\right\} \\
& <-\frac{1}{\eta} e^{-\eta(x+y)} \max \left\{1, \mathbb{E}\left[e^{-\eta(Y-y)}\right]\right\} .
\end{aligned}
$$

Thus $V_{0}(x)>V_{G}(x)$ for all non-zero fair gambles $G$, and it is never optimal for the agent with the timing option to gamble.

Now suppose that $u$ exhibits increasing absolute risk aversion. Then there is a direct analog of Lemma 1 for which we have that if $Y$ is advantageous at wealth $x$, then it is also advantageous for all smaller wealths, and then if $Q\left(x^{*}\right)=0$ we have $Q^{\prime}\left(x^{*}\right)<0$. It follows that if $Y$ is sometimes advantageous, then if the agent has initial wealth $x^{*}$ and if she enters the gamble paying $\epsilon Z$ and sells immediately if the gamble is successful, and waits if $Z=-1$, then she can improve her expected utility by gambling.

Finally suppose that the agent's absolute risk aversion is not monotonic, but has regions where it is locally decreasing or increasing. Then provided $Y-y$ is sufficiently small we have that the risk aversion is monotonic over the entire relevant range of outcomes, and the agent can gain from gambling. In particular, for fixed $u$ (provided it is not CARA) it is always possible to design a return distribution $Y$ such that the agent benefits from gambling.

\subsection{A numerical example in the one-period model}

Suppose $u(x)=\ln (x)$ and that $Y$ is a Bernoulli random variable which takes values $y u$ with probability $q$ and $y d$ with probability $1-q$ where $d<1<u$. By scaling, without loss of generality we may take $y=1$. Figure 1 considers the problem for the example $d=1 / 2, u=4$ and $q=3 / 4$. For these parameter values $Y$ is sometimes advantageous, which can be seen from the fact that the curves $\ln (x+y)$ and $\mathbb{E} \ln (x+Y)$ in the figure intersect at $x^{*}=-0.4905$. (Although cash wealth is negative at this point, the value of the agent's assets $x^{*}+y$ is positive.) Since $u(x)=\ln (x)$ is DARA, for initial wealths below $x^{*}$ the agent sells immediately, otherwise, she delays the sale. In the absence of the gamble, her maximum expected utility $V_{0}(x)$ is given by the solid line. For any initial wealth between $\alpha=-0.8066$ and $\beta=-0.4496$ the agent can improve her expected utility by gambling. For instance, if the agent's initial wealth is -0.6224 then the certainty equivalent value of the opportunity to gamble is 0.1238 . Thus the welfare gains are significant.

\section{The Asset Sale Problem in Continuous Time}

\subsection{The asset sale problem with no gambling opportunities}

In this section we will consider the case where the agent has no opportunities to enter independent gambles. In order to keep the formulation as simple as possible we assume that interest rates are zero, or equivalently that utility is measured with respect to discounted wealth. Furthermore 


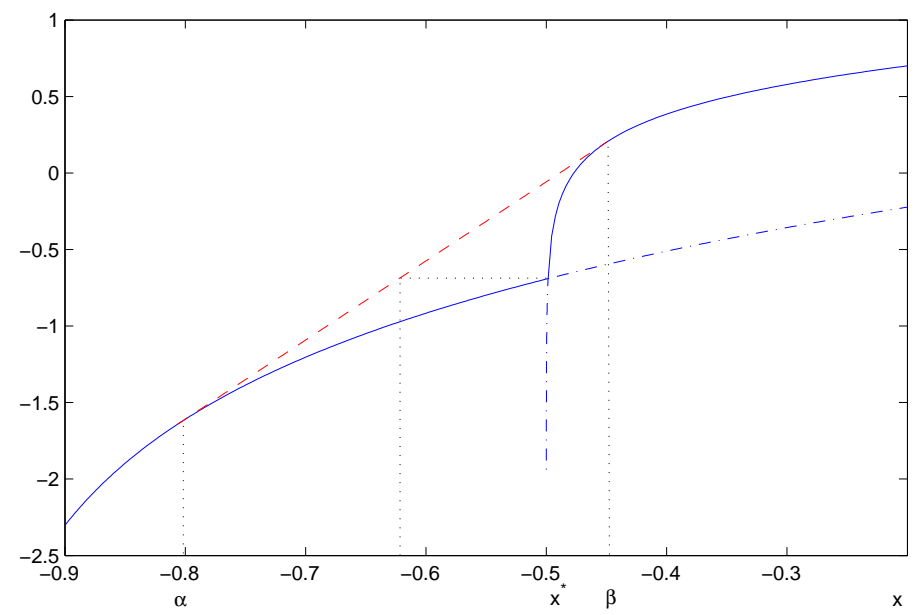

Figure 1: Value functions in the one period setting The graph uses $u(x)=\ln (x), y=1$ and a Bernoulli random variable $Y=4$ with probability $3 / 4$ and $Y=1 / 2$ otherwise. The solid line shows the value function $V_{0}(x)$ in the absence of the opportunity to gamble as a function of initial wealth. (Also shown are the value functions from following the strategies of selling now, and selling later, in the regions where these strategies are not optimal.) $V_{0}(x)$ is not concave, as can clearly be seen. The smallest concave function which dominates $V_{0}(x)$ is obtained by modifying the function to be given by the dashed line between $\alpha$ and $\beta$ where $(\alpha, \beta)=(-0.8066,-0.4496)$. This function represents the value function of an agent who at time zero is given a one-off opportunity to undertake a gamble of her choosing. 
we assume that utility is logarithmic ${ }^{8}$ and that the asset value $Y$ has log-normal dynamics. The price process $Y=\left(Y_{t}\right)_{t \geq 0}$ follows

$$
d Y_{t}=Y_{t}\left(\sigma d W_{t}+\mu d t\right)
$$

with volatility $\sigma$ and drift $\mu$. Since we assume zero interest rates, $\mu$ is also the risk premium on $Y$. This equation has solution $Y_{t}=y \exp \left\{\sigma W_{t}+\left(\mu-\sigma^{2} / 2\right) t\right\}$, where $Y_{0}=y$. Note that if $\mu>\sigma^{2} / 2$ then $Y_{t}$ grows to $\infty$ whereas, if $\mu<\sigma^{2} / 2$, then $Y_{t}$ tends to zero, almost surely. Set $\gamma=2 \mu / \sigma^{2} ; \gamma$ will play a key role in future calculations.

Consider an agent wishing to sell an indivisible asset at a time of her choosing and over an infinite horizon. Whilst she retains ownership of the asset the agent faces unhedgeable risk. She can remove this risk by selling, at the cost of forgoing the potential increase in value.

The problem facing the agent is to choose the sale time $\tau$ to maximize the expected utility of total wealth, or in other words to find

$$
V^{0}(x, y)=\sup _{\tau} \mathbb{E}\left[u\left(x+Y_{\tau}\right) \mid Y_{0}=y\right],
$$

where $u$ is logarithmic utility, $u(x)=\ln x$. The superscript 0 on the value function refers to the fact that we are working in the zero-gamble setting.

The problem (5) is amenable to classical optimal stopping techniques (Fleming and Rishel (1975)). The optimal stopping rule is of threshold form - stop the first time that the ratio of the asset price to wealth exceeds some level $z$. Thus we consider stopping times of the form

$$
\tau_{z}=\inf \left\{u: Y_{u} \geq x z\right\}
$$

Proposition 4 For $\gamma \leq 0, V^{0}(x, y)=\ln (x+y)$, and for $\gamma \geq 1, V^{0}(x, y)=\infty$.

In the non-degenerate case $0<\gamma<1$, in the selling region $y \geq x z^{*}$ we have $V^{0}(x, y)=$ $\ln (x+y)$, and in the waiting region $y<x z^{*}$,

$$
V^{0}(x, y)=\ln x+\left(\frac{y}{x z^{*}}\right)^{1-\gamma} \ln \left(1+z^{*}\right) .
$$

The optimal ratio $z^{*}=z^{*}(\gamma)$ is the unique solution in $(0, \infty)$ to $\Lambda^{\gamma}(z)=0$ where

$$
\Lambda^{\gamma}(z)=(1-\gamma) \ln (1+z)-\frac{z}{1+z}
$$

Proof: A formal proof of the proposition follows on checking that $V^{0}$ is a supermartingale under any stopping rule, and a true martingale under the stopping rule $\tau_{z^{*}}$. This proof also verifies that the optimal stopping rule is of the form presupposed above, namely to sell the asset the first time its price process exceeds $x z^{*}$. The details are supplied in the Appendix.

\subsection{The asset sale problem with a single gambling opportunity}

In this section we show that for some parameter values an agent with logarithmic utility can improve her expected utility by gambling. We restrict the agent to a single gamble to be taken at time zero.

\footnotetext{
${ }^{8}$ The results of the paper generalize to CRRA utility. However, the essential conclusions can be made for logarithmic utility so we present the results for that case.
} 
For a univariate function $v=v(\cdot)$, let $\bar{v}=\bar{v}(\cdot)$ denote the smallest concave majorant of $v$. Then $\bar{v}(x)=\sup _{\{G: \mathbb{E}[G]=0\}} \mathbb{E}[v(x+G)]$. More generally, for a bivariate function $v=v(x, y)$ we can consider $v$ as a family of univariate functions $v(\cdot, y)$ indexed by $y$. Then, for each fixed $y$ we let $\bar{v}(\cdot, y)$ denote the smallest concave majorant of $v(\cdot, y)$, thought of as a function of $x$.

Write $V^{1}(x, y)$ for the value function of the agent who, in addition to choosing the sale time $\tau$ is also free to enter into any fair gamble of her choosing at time zero. Then

$$
V^{1}(x, y)=\sup _{G: \mathbb{E}[G]=0} \mathbb{E}\left[V^{0}(x+G, y)\right]=\overline{V^{0}}(x, y) .
$$

The key result is that for certain regimes $V^{1}(x, y)>V^{0}(x, y)$.

Definition 5 Let $\Lambda^{\gamma}$ be as given in (7) and let $\gamma_{-}$be the unique solution in $(0,1)$ of $\Lambda^{\gamma}((1-$ $\left.\gamma)^{-1}\right)=0$. Then $\gamma_{-}$solves

$$
\left(1-\gamma_{-}\right)\left(2-\gamma_{-}\right) \ln \left(\frac{2-\gamma_{-}}{1-\gamma_{-}}\right)=1
$$

so that $\gamma_{-} \sim 0.3492$.

Theorem 6 For $\gamma \leq \gamma_{-}$there are no fair gambles such that the agent can improve her expected utility by entering the gamble. However, for $\gamma_{-}<\gamma<1$ there is a gamble such that the agent can improve her expected utility by entering this gamble.

Proof: The key step is to decide when the value function derived in Proposition 4 is concave in cash wealth $x$, for fixed $y .{ }^{9}$ It can be checked by direct calculation that $V^{0}(x, y)$ is concave in $x$ for all $x$ if $\gamma<\gamma_{-}$. Conversely, for $\gamma_{-}<\gamma<1$ the value function has positive second derivative in an interval to the right of $x=y / z^{*}$, see Figure 2. For a value of wealth in this interval (and more generally for a range of values of wealth including those in this interval) the agent can improve her expected utility (compared with the strategy of keeping cash wealth constant) by entering a suitable gamble. See the Appendix for more details.

\subsubsection{A numerical example in the continuous time model with a single gamble}

Recall $u(x)=\ln (x)$ and take $\gamma=0.75>\gamma_{-}$. By scaling, without loss of generality we may take $y=1$. Figure 2 shows the value function $V^{0}(x, 1)$, as given in Proposition 4 , where, for initial wealths below $w^{*}=1 / z^{*}=0.0202$, the agent sells immediately, and otherwise waits. The agent can improve her expected utility by gambling for any initial wealth between $\alpha$ and $\beta$ where $(\alpha, \beta)=(-0.4626,0.2589)$. (The smallest concave majorant $\overline{V^{0}}(x, 1)$ which dominates $V^{0}(x, 1)$ is obtained by modifying the function to be given by the dashed line between $\alpha$ and $\beta$. This function represents the value function $V^{1}(x, 1)$ of an agent who at time zero is given a one-off opportunity to undertake a gamble of her choosing.) In fact, if the agent's initial wealth is -0.1181 , then the certainty equivalent value of a one-off opportunity to gamble at time 0 is 0.1383 . This is clearly non-trivial when compared to the other parameters, and so there are substantial welfare gains to be had by accessing gambling opportunities. Of course, she may do even better by taking a series of gambles over time and we will show this in the following section.

\footnotetext{
${ }^{9}$ Note that for a fixed $x$ the value function of the agent who cannot gamble is always concave in $y$.
} 


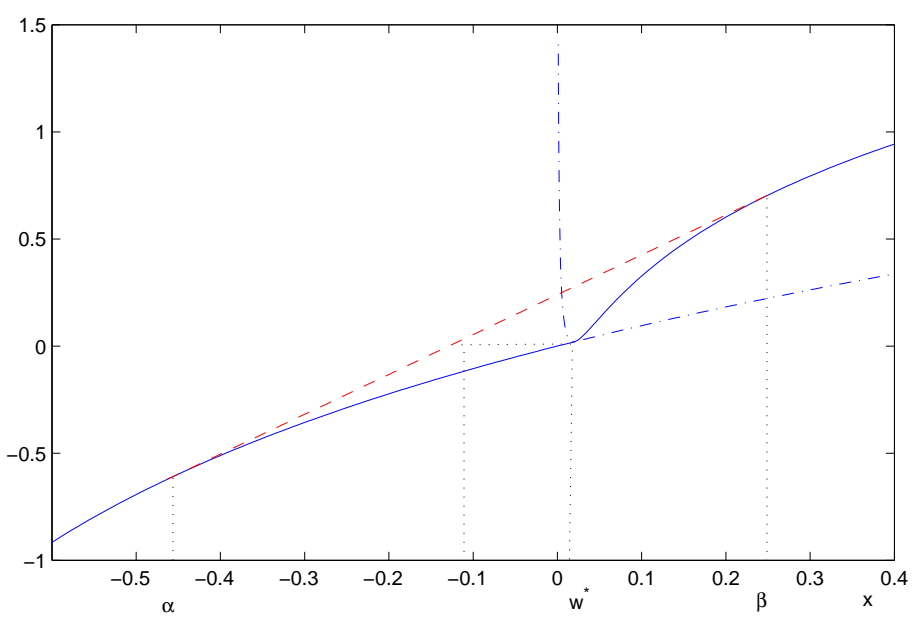

Figure 2: The value function for the agent without access to gambles. The graph uses values $y=1$ and $\gamma=0.75$. The solid curve on the graph represents the value function $V^{0}(x, 1)$, as given in Proposition 4. To the left of $w^{*}=1 / z^{*}=0.0202, V^{0}(x, 1)$ is given by $\ln (1+x)$, and to the right of $w^{*}, V^{0}(x, 1)$ is given by (6). Also shown are the continuations of these functions into the domains where they are not relevant. Since $0.75>\gamma_{-}, V^{0}(x, 1)$ is not concave. The smallest concave majorant $\overline{V^{0}}(x, 1)$ which dominates $V^{0}(x, 1)$ is obtained by modifying the function to be given by the dashed line between $\alpha$ and $\beta$ where $(\alpha, \beta)=(-0.4626,0.2589)$. This function represents the value function $V^{1}(x, 1)$ of an agent who at time zero is given a one-off opportunity to undertake a gamble of her choosing. The certainty equivalent value of the opportunity to gamble for an agent with initial wealth -0.1181 is 0.1383 . 


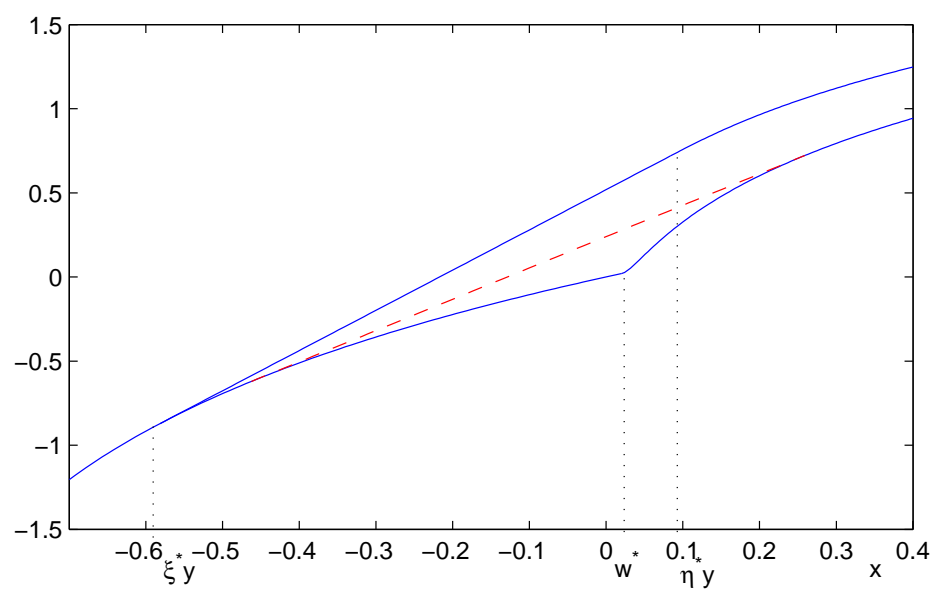

Figure 3: The value function for the agent with access to repeated gambles. The graph uses values $y=1$ and $\gamma=0.75$. The values of $\eta^{*}$ and $\xi^{*}$ are 0.084 and -0.582 respectively. The higher solid curve on the graph represents the value function $V^{\infty}(x, 1)$, as given in Theorem $\%$. To the left of $-0.582, V^{\infty}(x, 1)$ is given by $\ln (1+x)$, between -0.582 and $0.084, V^{\infty}(x, 1)$ is given in (14), and to the right of $0.084, V^{\infty}(x, 1)$ is given in (15).

\subsection{The asset sale problem with repeated gambling opportunities}

In this section we revisit the optimal asset sale problem in a setting where the agent has outside investment opportunities. The asset price $Y$ follows the dynamics given in (4). In addition, there is a traded asset with price process $P=\left(P_{t}\right)_{t \geq 0}$ where $P$ follows exponential Brownian motion

$$
d P_{t}=P_{t} \psi d B_{t}
$$

where $B=\left(B_{t}\right)_{t \geq 0}$ is a Brownian motion independent of $W$. The traded asset $P$ has two key properties: it is independent of the asset $Y$, and it has zero drift and hence zero Sharpe ratio. Given these properties, a position in $P$ has a natural interpretation as taking a fair gamble. (Obviously if these properties did not hold, then it would not be surprising that the agent could improve her expected utility either by holding a position in $P$ to benefit from a positive Sharpe ratio, or to offset some of the risk of $Y$ by hedging in a correlated $P$ ).

The agent can choose strategy $\pi=\left(\pi_{t}\right)_{t \geq 0}$ to obtain trading wealth $X=X^{\pi}=\left(X_{t}^{\pi}\right)_{t \geq 0}$ where $X^{\pi}$ solves

$$
d X_{t}^{\pi}=\pi_{t} \frac{d P_{t}}{P_{t}}=\pi_{t} \psi d B_{t}
$$

The problem facing the agent is to choose the sale time $\tau$ and the investment strategy $\pi$ over $[0, \tau]$ to maximize expected utility of total wealth, or in other words to find

$$
V^{\infty}(x, y)=\sup _{\tau} \sup _{\pi} \mathbb{E}\left[u\left(X_{\tau}^{\pi}+Y_{\tau}\right) \mid X_{0}=x, Y_{0}=y\right]
$$

where $u(x)=\ln (x)$ and the superscript infinity on $V$ denotes that the agent can trade an infinite number of times. The Hamilton-Jacobi-Bellman equation for the problem gives us

$$
\max \left\{\ln (x+y)-V^{\infty}(x, y), V_{x x}^{\infty}, \gamma y V_{y}^{\infty}+y^{2} V_{y y}^{\infty}\right\}=0
$$


with value matching and smooth fit at the boundaries. The three elements of this equation correspond to the exercise region, the gambling region and a waiting/continuation region in which the investment in the traded asset is zero.

Recall the definitions of $z^{*}(\gamma)$ and $\gamma_{-}$from Sections 2.1 and 2.2. For $\gamma_{-}<\gamma<1$ define

$$
\begin{gathered}
\eta^{*}=\eta^{*}(\gamma)=\frac{1-\gamma}{2-\gamma}\left(\frac{1}{1-\gamma}-\ln \left(\frac{2-\gamma}{1-\gamma}\right)\right)^{-1} \\
\xi^{*}=\xi^{*}(\gamma)=\frac{\eta^{*}(2-\gamma)}{1-\gamma}-1=\frac{(1-\gamma) \ln ((2-\gamma) /(1-\gamma))-\gamma}{1-(1-\gamma) \ln ((2-\gamma) /(1-\gamma))}
\end{gathered}
$$

and

$$
\Theta^{*}=\frac{1}{(1-\gamma)(2-\gamma)}
$$

It can be shown that $-1<\xi^{*}(\gamma)<1 / z^{*}(\gamma)$ and $\eta^{*}(\gamma)>\max \left\{0, \xi^{*}(\gamma)\right\}$.

We prove the following theorem in the Appendix.

Theorem 7 (i) For $\gamma \leq 0, V^{\infty}(x, y)=\ln (x+y)$.

(ii) For $0<\gamma \leq \gamma_{-}$, the value function in the selling region $y \geq x z^{*}$ is given by $V^{\infty}(x, y)=$ $\ln (x+y)$ and in the continuation region $y<x z^{*}$ is given by

$$
V^{\infty}(x, y)=\ln x+\left(\frac{y}{x z^{*}}\right)^{1-\gamma} \ln \left(1+z^{*}\right)
$$

(iii) For $\gamma_{-}<\gamma<1$ the value function in the selling region $x \leq y \xi^{*}$ is given by $V^{\infty}(x, y)=$ $\ln (x+y)$; in the gambling region $y \xi^{*}<x<y \eta^{*}$ is given by

$$
V^{\infty}(x, y)=\frac{\xi^{*}}{\eta^{*}-\xi^{*}}\left(\frac{x}{\xi^{*} y}-1\right)\left(\ln y+\ln \eta^{*}+\Theta^{*}\right)+\frac{\eta^{*}}{\eta^{*}-\xi^{*}}\left(1-\frac{x}{\eta^{*} y}\right)\left(\ln y+\ln \left(1+\xi^{*}\right)\right)
$$

and in the waiting/continuation region $x \geq y \eta^{*}$ is given by

$$
V^{\infty}(x, y)=\ln x+\left(\frac{\eta^{*} y}{x}\right)^{1-\gamma} \Theta^{*}
$$

where $\eta^{*}(\gamma), \xi^{*}(\gamma)$ and $\Theta^{*}$ are given by (10), (11) and (12).

Corollary 8 For $\gamma \leq \gamma_{-}$there is no non-zero investment strategy $\pi$ such that the agent can improve her expected utility and $V^{\infty}(x, y)=V^{0}(x, y)$. However, for $\gamma_{-}<\gamma<1$ the agent can improve her utility via investing in the asset $P$, akin to taking fair gambles. In this case her expected utility is greater than that she can attain from a single gamble at time zero.

\subsubsection{A numerical example in the continuous time model with repeated gambling}

Recall $u(x)=\ln (x)$ and take $y=1, \gamma=0.75>\gamma_{-}$. Then $\eta^{*}=0.084, \xi^{*}=-0.582$ and $\Theta^{*}=3.2$. Again, by scaling we may set $y=1$. Figure 3 shows the value function $V^{\infty}(x, 1)$, as given in Theorem 7 , where $V^{\infty}(x, 1)=\ln (x+y)$ for $x \leq-0.582 ; V^{\infty}(x, 1)$ is given by (14) for $-0.582<x<0.084$; and $V^{\infty}(x, 1)$ is given by (15) for $x \geq 0.084$. Also shown is the value function $V^{0}(x, 1)$ and the single gamble described in the example of Section 2.2.1. It is clear from the figure that the agent has higher expected utility with access to repeated gambling than for a single gamble.

In the common stopping region, $x \leq y \xi^{*}$ we have that $V^{\infty}(x, y)=V^{1}(x, y)=V^{0}(x, y)$, but for $x>\eta^{*} y$ we have $V^{\infty}(x, y)>V^{0}(x, y)$ even though in both cases the optimal policy is to do nothing immediately. In this case the agent who can trade in $P$ may take advantage of this opportunity in the future, and this gives her extra value. 


\section{Alternative Explanations of Gambling}

As mentioned in the introduction there are many instances and explanations of gambling from the economics, finance and decision theory literatures. We review these alternative explanations, and argue that the phenomena which leads to gambling in this article is both distinct and original.

For the majority of alternative rationalizations of gambling, either the agents are not maximizers of expected utility, or their utility function is not concave. In the former class we include agents who receive utility from the act of gambling and agents who are overconfident, and who miscalculate the probabilities of events (and especially events with small probabilities). In the latter class we include reference levels and $S$-shaped utility functions, and other situations in which the agent has explicit non-concave objectives. These models provide justifications and explanations for gambling, but the conclusion that risk-seeking behavior is optimal is often by design, and is not unexpected.

Examples of a non-concave objective are to be found in the literature on option compensation for managers, and shareholders with limited liability. Let $Y$ be the price of an underlying asset, $f$ a payoff function, and $u$ a utility. The properties of the indirect utility $v(x, y)=\mathbb{E}[u(x+f(Y))]$ as a function of price $y$, clearly depend on the combined effect of $u$ and $f$. If $u$ is linear and $f$ convex (such as a call option) then we have the well-known gambling for resurrection phenomena ${ }^{10}$. If $u$ is concave and $f$ is convex, then for fixed wealth $x, v$ may be both locally convex and concave in price $y$. Ross (2004) (see also Carpenter (2000) in continuous time) study the combination of concave utilities and convex payoffs to determine whether risk-taking behavior (in terms of desiring price volatility) is induced when risk averse managers receive option compensation. In contrast, our study is concerned with properties of $V^{0}(x, y)$ as a function of wealth, $x$. In particular, any gambling takes place in other outside assets rather than the asset $Y$ itself. In fact, in our set-up, $V^{0}(x, y)$ is concave everywhere in $y$, and thus there are no price-risk taking incentives at all. Importantly, intuition from the compensation literature concerning risk taking in the price dimension cannot be simply transferred across to our problem, and vice versa. For instance, our outside gambling is not universally attractive but depends on preferences. In particular, whilst price-risk taking (of Ross (2004) or Carpenter (2000)) can occur for CARA, outside gambling will not. Further, price-risk taking can occur when the payoff $f(Y)$ is to be received at a fixed time, whilst outside gambling requires a choice over when to receive the payout. (Note our gambling is applicable in most real options and compensation problems which typically involve indivisible American style payoffs). Finally, since it is easier to envisage agents having mechanisms to take gambles in other assets but not being able to influence the price $Y$ itself (through its volatility), our finding is more widely applicable than the price-risk taking results.

At an abstract level, the mechanism that leads to gambling in the price-risk literature is the existence of a non-concave objective function. The source of gambling in our model is more subtle and there are no non-concavities in the model set-up. Instead, and again at the abstract level, the convexity in our problem arises from the fact that the optimization problem for the agent involves a maximization over a control, and the fact that the maximization operator is not concavity preserving (the maximum of a set of concave functions need not be concave). This phenomena has arisen before in the literature, but always in different context, for example in

\footnotetext{
${ }^{10}$ The limited liability of shareholders gives them an incentive to "gamble for resurrection" when faced with bankruptcy (Jensen and Meckling (1976)). If the firm value can jump into bankruptcy (as is the case in a one period model and some continuous time models with jumps), then since equity value is a convex function of firm value, shareholders will prefer to maximize volatility.
} 
the literature on profit maximizers preferring price uncertainty (see Hartman (1972) and Varian (1992) p43, and in continuous time, Abel (1983) and Pindyck (1982)). A further example is the literature on consumption commitments, see Postlewaite, Samuelson and Silverman (2008). For example, let $\hat{f}$ be a production function, $P$ the (random) price of a good and $\pi$ the production level (a variable under the control of an agent), and suppose that the profit to the agent is given by

$$
\hat{U}(p, \pi)=\hat{f}(\pi)-\pi p
$$

We have that $\hat{U}$ is linear in $p$ but the profit at the optimum production

$$
\hat{V}(p):=\max _{\pi} \hat{U}(p, \pi)
$$

is not; for example if $\hat{f}(\pi)=2 \sqrt{\pi}$ then $\hat{V}(p)=1 / p$ which is globally convex. It follows that the value function of the profit maximizing agent is such that $\mathbb{E}[\hat{V}(P)] \geq \hat{V}(\mathbb{E}[P])$ and the agent prefers price uncertainty.

Returning to our agent with the timing option (in the one-period model), then if by analogy with (16) and (17) we write

$$
U(x, \pi)=\mathbb{E}[u(x+y+\pi(Y-y))]
$$

where $\pi$ represents the holding of the asset $Y$ over time period $(0,1)$, then

$$
v(x)=\max _{\pi \in A} U(x, \pi)
$$

is concave for any concave $u$ provided the set $A$ is an interval. To see this suppose $\lambda \in(0,1)$ and let $\tilde{x}=\lambda x_{1}+(1-\lambda) x_{2}$. For $i=1,2$ let $\pi_{i}$ be the optimal choice of scale for wealth $x_{i}$ and let $\tilde{\pi}=\lambda \pi_{1}+(1-\lambda) \pi_{2}$; then $\tilde{\pi} \in A$ by the convexity of $A$. Then

$$
\begin{aligned}
v(\tilde{x}) & \geq \mathbb{E}[u(\tilde{x}+\tilde{\pi} Y+(1-\tilde{\pi}) y)] \\
& =\mathbb{E}\left[u\left(\lambda\left(x_{1}+\pi_{1} Y+\left(1-\pi_{1}\right) y\right)+(1-\lambda)\left(x_{2}+\pi_{2} Y+\left(1-\pi_{2}\right) y\right)\right)\right] \\
& \geq \lambda \mathbb{E}\left[u\left(x_{1}+\pi_{1} Y+\left(1-\pi_{1}\right) y\right)\right]+(1-\lambda) \mathbb{E}\left[u\left(x_{2}+\pi_{2} Y+\left(1-\pi_{2}\right) y\right)\right] \\
& =\lambda v\left(x_{1}\right)+(1-\lambda) v\left(x_{2}\right),
\end{aligned}
$$

and $v$ is concave. In our case the key point is the indivisibility of the asset. This means that the choice is taken over the non-convex set $A=\{0,1\}$ corresponding to the choice to sell the whole unit now or later. Thus, although the convexity can only occur in our model because of the optimization over the (timing) choice variable, a further requirement is that the asset is indivisible, so that the set of admissible controls is not convex. This requirement distinguishes the mechanism present in our analysis from the price-uncertainty literature.

Finally, we mention a paper by Kwang (1965) in which indivisibility is key to gambling behavior. There the agent with wealth $x$ has the option to pay $c$ to receive a fixed utility $k$. The agent's utility is then given by

$$
v(x)=\max \{u(x), u(x-c)+k\}
$$

(This can be represented by a diagram which is qualitatively very similar to Figure 1, and again gambling is optimal near the point of indifference.) However, there is no way that the option to pay $c$ to receive a fixed increase in utility of size $k$ (independently of current wealth) can be reconciled with decreasing marginal utility of wealth, and so the analysis cannot be made 
consistent with an expected utility framework. Although indivisibility is key to the gambling in the analysis of Kwang, it is difficult to disentangle from the non-concavity in the objective function.

In the absence of the asset to sell it is never optimal for an agent with increasing, strictlyconcave von Neumann-Morgenstern preferences to enter into a fair gamble, in the sense that to do so reduces her expected utility. By an application of the conditional Jensen's inequality the same remains true if the agent owns the asset, but cannot sell until a later time. In this case continued ownership of the asset is equivalent to what is known in the literature as a background risk, and it is well known that a fair gamble cannot be made attractive by the presence of a background risk. Conventional wisdom suggests that stronger statements on preferences are also true, and that bearing one risk makes an agent less willing to bear another independent risk. However, not all preferences are consistent with this stronger statement, and hence there has been a program in the literature - Proper risk aversion (Pratt and Zeckhauser (1987)), Standard risk aversion (Kimball (1993)), Risk vulnerability (Gollier and Pratt (1996)) - to determine additional conditions on utility functions which are equivalent to some notion of the idea that adding a gamble can never make an unattractive risk into an attractive one. The set-up of this paper is different in that we are interested in the impact of adding a fair gamble to a situation where the an agent has a choice over whether to accept a background risk (or in our terminology, whether to sell now or later). We show that even though the agent is risk averse, she may be able to increase her expected utility by entering into an independent gamble which has zero expectation.

\section{Further Remarks and Conclusions}

The main goal of the paper has been to argue that a risk averse agent with a timing option over when to sell an asset may benefit from gambling, provided that she can delay the decision to sell until the result of the gamble is known. The key point is that even if the expected utility of selling now and selling later may be the same, the marginal utility (with respect to wealth) will, in general, be different. This difference in marginal utility leads to the convexity which the agent can exploit by gambling. Put another way, the value function is the maximum of two concave functions, and this need not be concave.

Since the principles which lead to the convexity are so general, it means that this phenomena should be widespread. It does however, rely on several key components of the set-up. Firstly, the asset must be appreciating in value, but not so much that the return always outweighs the risk. Secondly, the market must be incomplete. If the market is complete then the agent can remove the risk associated with delaying sale via a hedge. Thirdly, it is clear that the agent can only benefit from the gamble if she can make the decision to sell contingent upon the outcome of the gamble. This ordering of the decisions is key to the single period model, although it is a natural feature of any multi-period or the continuous time model. Finally, the asset must be indivisible. If the agent can choose the scale of her investment then the potential benefits from a gamble disappear. All of these features may be present in asset sale (and real option) decisions, executive compensation, as well as in less obvious contexts such as the decision to stop or continue a political campaign, or whether to return to college.

It remains to comment on the differences between the discrete and continuous models. In the discrete setting it is the case that, provided that $\mathrm{Y}$ is sometimes advantageous (and not never advantageous or always advantageous), then except in a small number of pathological cases, and except in the special case of CARA utility, the agent can always benefit from a gamble, prior to taking the decision over whether to sell now or later. If the utility exhibits DARA then the 
optimal strategy is to delay sale if the fair gamble has a positive outcome.

In the continuous time setting the story is more delicate. We have shown that there is a natural set-up (logarithmic utility and lognormal asset prices such that the asset is sometimes advantageous) in which the agent who is indifferent between selling and waiting can benefit from a gamble. But we have also shown that for other parameter values (such that the asset is again sometimes advantageous) there is no benefit to be gained from gambling. This is in distinct contrast to the discrete case in which there are always benefits from gambling if the asset is sometimes advantageous. The reason for this difference is the smoothing effect of the timing decision in continuous time. Since the agent is maximizing over many (path-dependent) stopping rules, (rather than just two choices in the discrete time model) we are taking the maximum over a family of concave functions. If this family is sufficiently broad, then the resulting value function may be concave.

\section{$5 \quad$ References}

Abel, A.B. (1983): "Optimal Investment Under Uncertainty," American Economic Review, 73, 228-233.

Arrow, K.J. (1971): Essays in the Theory of Risk Bearing, Chicago: Markham.

Barberis N. and W. Xiong (2011): "Realization Utility", Journal of Financial Economics, forthcoming.

Basak, S., Pavlova, A. and A. Shapiro (2007): "Optimal Asset Allocation and Risk Shifting in Money Management," Review of Financial Studies, 20, 1583-1621.

Brennan, M.J. and E. Schwartz, (1985): "Evaluating Natural Resource Investments," Journal of Business, 58, 135157.

Campbell, J.Y. and L.M. Viceira (2002): Strategic Asset Allocation: Portfolio Choice for Long-Term Investors, Oxford University Press.

Carpenter, J.N. (2000): "Does Option Compensation Increase Managerial Risk Appetite?," Journal of Finance, 55, 2311-2331.

Carpenter, J.N., Stanton, R. and N. Wallace (2010): "Optimal Exercise of Executive Stock Options and Implications for Firm Cost," Journal of Financial Economics, 98, 315-337.

Décamps, J.P and T. Mariotti (2004): "Investment Timing and Learning Externalities," Journal of Economic Theory, 118, 80-102.

Décamps, J.P., T. Mariotti, and S. Villeneuve (2005): "Investment timing under incomplete information", Math. Oper. Res., 30, 472500.

Detemple J. and S. Sundaresan (1999): "Nontraded Asset Valuation with Portfolio Constraints: A Binomial Approach", Review of Financial Studies, 12(4), 835-872.

Dixit, A.K. and R.S. Pindyck (1994): Investment under Uncertainty, Princeton University Press.

Dybvig, P.H. and Lippman, S.A. (1983): "An Alternative Characterization of Decreasing Absolute Risk Aversion," Econometrica, 51, 223-224.

Evans, J., Hobson D., and V. Henderson (2008): "Optimal Timing for an Indivisible Asset Sale." Mathematical Finance, 18, 545-567.

Fleming, W.H. and R.W. Rishel (1975): Deterministic and Stochastic Optimal Control, SpringerVerlag.

Gollier, C. and J.W. Pratt (1996): "Risk Vulnerability and the Tempering Effect of Background Risk," Econometrica, 64, 1109-1123. 
Guo, X., Miao, J. and E. Morellec (2005): "Irreversible Investment with Regime Shifts", Journal of Economic Theory, 122, 37-59.

Hartman, R. (1972): "The Effects of Price and Cost Uncertainty on Investment," Journal of Economic Theory, 5, 258-66.

Hall, B.J. and K.J. Murphy (2000): "Optimal Exercise Prices for Executive Stock Options," American Economic Review, 90, 209-214.

Henderson, V. (2007): "Valuing the Option to Invest in an Incomplete Market," Mathematics and Financial Economics, 1, 103-128.

Hugonnier, J. and E. Morellec (2007): "Corporate Control and Real Investment in Incomplete Markets," Journal of Economic Dynamics and Control, 31, 1781-1800.

Ingersoll, J. (2006): "The Subjective and Objective Evaluation of Incentive Stock Options," Journal of Business, 79, 453-487.

Jensen M.C. and W.H. Meckling (1976): "Theory of the Firm: Managerial Behavior, Agency Costs and Ownership Structure", Journal of Financial Economics, 3, 305-360.

Kahl M. Liu J. and F.A. Longstaff (2003): "Paper Millionaires: How Valuable is Stock to a Stockholder who is Restricted from Selling it?", Journal of Financial Economics, 67, 385-410.

Kahneman, D. and A. Tversky (1979): "Prospect Theory: An Analysis of Decision Under Risk," Econometrica, 46, 171-185.

Karatzas I. and H. Wang (2000): "Utility Maximization with discretionary stopping", SIAM J.Control and Optim., 39, 306-329.

Kimball, M.S. (1993): "Standard Risk Aversion," Econometrica, 61, 589-611.

Kwang, N.Y. (1965): "Why Do People Buy Lottery Tickets? Choices Involving Risk and the Indivisibility of Expenditure," Journal of Political Economy, 73, 530-535.

Kwon H.D. (2010): "Invest or Exit? Optimal Decisions in the Face of a Declining Profit Stream", Operations Research, 58(3), 638649.

Leland, H.E.(1998): "Agency Costs, Risk Management and Capital Structure," Journal of Finance, 53, 1213-1243.

Leung T. and R. Sircar (2009): "Accounting for risk aversion, vesting, job termination risk and multiple exercises in valuation of employee stock options", Mathematical Finance, 19, 99-128.

McDonald, R. and D.R. Siegel (1986): "The Value of Waiting to Invest," Quarterly Journal of Economics, 101, 707-727.

McKean, H.P. (1965): "A Free Boundary Problem for the Heat Equation Arising from a Problem in Mathematical Economics", Industrial Management Review, 6, Spring, 32-39. (Appendix to Samuelson (1965)).

Merton R.C. (1969): "Lifetime Portfolio Selection under Uncertainty: The Continuous Time Case," Review of Economics and Statistics, 51, 247-257.

Merton, R.C. (1973): "Theory of Rational Option Pricing," Bell Journal of Economics and Management, 4, 141-183.

Miao, J. and N. Wang (2007): "Investment, Hedging and Consumption Smoothing," Journal of Financial Economics, 86, 608-642.

Pindyck, R.S., (1982): "Adjustment Costs, Uncertainty, and the Behavior of the Firm," American Economic Review, 72, 415-427.

Postlewaite, A., Samuelson L. and D. Silverman (2008): "Consumption Commitments and Employment Contracts," Review of Economic Studies, 75, 559-578.

Pratt, J.W. (1964): "Risk Aversion in the Small and in the Large," Econometrica, 32, 122-136.

Pratt, J.W., and R.J. Zeckhauser (1987): "Proper Risk Aversion," Econometrica, 55, 143-154.

Ross, S.A. (2004): "Compensation, Incentives, and the Duality of Risk Aversion and Riskiness," Journal of Finance, 59, 207-225. 
Samuelson, P.A. (1965): "Rational Theory of Warrant Pricing," Industrial Management Review, 6, Spring, 13-31.

Smith J.E. and K.F. McCardle (1998): "Valuing Oil Properties: Integrating Option Pricing and Decision Analysis Approaches", Operations Research, 46(2): 198-217.

Smith J.E. and R.F. Nau (1995): "Valuing Risky Projects: Option Pricing Theory and Analysis", Management Science, 41(5), 795-816.

Varian, H.R. (1992): Microeconomic Analysis, Third Edition, W.W. Norton and Company, Inc.

\section{A Appendix: Proofs}

\section{Proof of Proposition 4}

We cover the case $0<\gamma<1$. Let $V^{c}$ be defined via $V^{c}(x, y)=\ln (x+y)$ for $y \geq x z^{*}$, and $V^{c}$ as given by the right-hand-side of (6) otherwise. The superscript $c$ refers to the fact that this is the conjectured value function. We aim to show that with this definition $V^{c} \equiv V^{0}$, where $V^{0}$ is the solution to the problem in (5).

We saw in the discussion before the statement of Proposition 4, that there is a stopping rule, namely $\tau_{z^{*}}=\inf \left\{u: Y_{u} \geq x z^{*}\right\}$, for which the value function is $V^{c}$. Hence $V^{c}$ is a lower bound on the value function for the optimal stopping problem. It remains to show that $V^{c}$ is an upper bound, and hence that $V^{c}=V^{0}$.

It is an exercise in one-dimensional calculus to show that $V^{c}$ satisfies (a subscript $y$ denotes partial differentiation)

$$
\begin{aligned}
V^{c}(x, y) & \geq \ln (x+y) \\
\mu y V_{y}^{c}+\frac{1}{2} \sigma^{2} y^{2} V_{y y}^{c} & \leq 0
\end{aligned}
$$

with equality in (A1) for $y \geq x z^{*}$, and equality in (A2) for $y<x z^{*}$.

By Ito's formula

$$
\begin{aligned}
V^{c}\left(x, Y_{t}\right) & =V^{c}(x, y)+\int_{0}^{t}\left(\mu Y_{s} V_{y}^{c}\left(x, Y_{s}\right)+\frac{1}{2} \sigma^{2} Y_{s}^{2} V_{y y}^{c}\left(x, Y_{s}\right)\right) d t+\int_{0}^{t} \sigma Y_{s} V_{y}^{c}\left(x, Y_{s}\right) d W_{s} \\
& \leq V^{c}(x, y)+\int_{0}^{t} \sigma Y_{s} V_{y}^{c}\left(x, Y_{s}\right) d W_{s} .
\end{aligned}
$$

The right hand side of this expression is a local martingale which is bounded below (by $V^{c}(x, 0)=$ $\ln x$ ) and hence is a super-martingale. Then, using (A1), for any stopping time $\tau$,

$$
\mathbb{E}^{y}\left[\ln \left(x+Y_{\tau}\right)\right] \leq \mathbb{E}^{y}\left[V^{c}\left(x, Y_{\tau}\right)\right] \leq V^{c}(x, y)+\mathbb{E}^{y}\left[\int_{0}^{\tau} \sigma Y_{s} V_{y}^{c}\left(x, Y_{s}\right) d W_{s}\right] \leq V^{c}(x, y)
$$

and $V^{c}$ is also an upper bound.

\section{Proof of Theorem 6}

Fix $\gamma \in(0,1)$ and $y>0$. For $x>y / z^{*}, V^{0}$ is as in (6), and using the definition of $z^{*}$,

$$
V_{x x}^{0}(x, y)=-\frac{1}{x^{2}}\left[1-\frac{(2-\gamma) z^{*}}{\left(1+z^{*}\right)}\left(\frac{y}{x z^{*}}\right)^{1-\gamma}\right]
$$


$V^{0}$ is concave in $x$ for all $x \geq y / z^{*}$, and hence concave everywhere, provided the term in square brackets is positive for $x>y / z^{*}$. Since $\left(y / x z^{*}\right)^{1-\gamma}$ is decreasing in $x$, this happens if

$$
1 \geq \frac{(2-\gamma) z^{*}}{\left(1+z^{*}\right)}
$$

or equivalently if $z^{*} \leq(1-\gamma)^{-1}$. If $z^{*}>(1-\gamma)^{-1}$ then $V^{0}$ is locally convex in $x$ over the region

$$
\frac{y}{z^{*}}<x<\frac{y}{z^{*}}\left(\frac{z^{*}(2-\gamma)}{\left(1+z^{*}\right)}\right)^{1 /(1-\gamma)} .
$$

It remains to check whether $z^{*}<(1-\gamma)^{-1}$.

If $\gamma>\gamma_{-}$then $\Lambda^{\gamma}\left((1-\gamma)^{-1}\right)<0$, and from the fact that $\Lambda^{\gamma}$ is positive for $z>z^{*}(\gamma)$ we conclude that $z^{*}(\gamma)>(1-\gamma)^{-1}$. Otherwise, for $\gamma<\gamma_{-}, V_{x x}^{0} \leq 0$ everywhere.

Fix $\gamma \in\left(\gamma_{-}, 1\right)$ and suppose $y=1$. Results for other values of $y$ can be obtained by scaling. We want to show that depending on the value of $x$, there may be a gamble the agent can use to improve her expected utility, when compared with a strategy of never gambling.

Let $\overline{V^{0}}(x, 1)$ be the smallest concave majorant of $V^{0}(x, 1)$, see Figure 2. Then $\overline{V^{0}}(x, 1)=$ $V^{0}(x, 1)$ for $x$ outside the region $(\alpha, \beta)$. For initial values of wealth in the interval $(\alpha, \beta)$, suppose the agent enters a gamble which either leaves the cash reserves at $\beta$ with probability $(x-\alpha) /(\beta-$ $\alpha$ ) or at $\alpha$ with probability $(\beta-x) /(\beta-\alpha)$, and suppose that after this gamble the agent enters no further gambles. Then her value function is given by

$$
\frac{(\beta-x)}{(\beta-\alpha)} V^{0}(\alpha, 1)+\frac{(x-\alpha)}{(\beta-\alpha)} V^{0}(\beta, 1) \equiv \overline{V^{0}}(x, 1)
$$

and $\overline{V^{0}}(x, 1)>V^{0}(x, 1)$. Note that we do not claim that this is the optimal strategy in general, but it is optimal if the agent is allowed a single gamble which must be taken immediately, but which may be constructed according to her own design, subject to the condition that it is fair.

\section{Proof of Theorem 7}

It is sufficient to find $V^{\infty}$ such that $V^{\infty}(x, y) \geq \ln (x+y)$ with equality in the selling region, and $V^{\infty}\left(X_{t}^{\pi}, Y_{t}\right)$ is a supermartingale in the continuation region and a martingale for the optimal $\pi$. Applying Itô's formula to $V^{\infty}$, and considering the drift term we conclude that

$$
\sup _{\pi}\left\{\frac{\pi^{2}}{2} \psi^{2} V_{x x}^{\infty}+\mu y V_{y}^{\infty}+\frac{\sigma^{2}}{2} y^{2} V_{y y}^{\infty}\right\}=0
$$

If $V_{x x}^{\infty}>0$ this leads to a contradiction. Hence we must have that $V_{x x}^{\infty} \leq 0$ and, if $V_{x x}^{\infty}<0$ then $\gamma y V_{y}^{\infty}+y^{2} V_{y y}^{\infty}=0$.

If $0<\gamma<\gamma_{-}$, then the expression in (13) solves $\gamma y V_{y}^{\infty}+y^{2} V_{y y}^{\infty}=0$ and $V_{x x}^{\infty}<0$ on $y<x z^{*}$. Hence (9) is satisfied.

If $\gamma_{-}<\gamma<1$ then (13) is not the solution of (9) since $V_{x x}^{\infty}<0$ does not hold. Instead, we look for a solution such that $V^{\infty}(x, 0)=\ln x$ and

$$
\begin{aligned}
V^{\infty}(x, y)=\ln (x+y) ; & x \leq \xi y \\
V_{x x}^{\infty}=0 ; & \xi y<x<\eta y \\
\gamma y V_{y}^{\infty}+y^{2} V_{y y}^{\infty}=0 ; & x \geq \eta y
\end{aligned}
$$


and such that there is value matching and smooth fit at $x=\xi y$ and value matching and smooth fit to second order at $x=\eta y$.

In the region $x \geq \eta y$ we have $V^{\infty}(x, y)=\ln x+\Theta\left(\frac{\eta y}{x}\right)^{1-\gamma}$ for some unknown $\Theta$. For $\xi y<$ $x<\eta y$ we have

$$
\begin{aligned}
V(x, y) & =V(\xi y, y)+\{V(\eta y, y)-V(\xi y, y)\} \frac{(x-\xi y)}{(\eta-\xi) y} \\
& =\ln y+\ln (1+\xi)+\{\ln \eta+\Theta-\ln (1+\xi)\} \frac{(x-\xi y)}{(\eta-\xi) y}
\end{aligned}
$$

Using smooth fit (ie. continuity of $V_{x}$ ) at $x=\xi y$ and $x=\eta y$ we have

$$
\frac{1}{(1+\xi)}=\frac{1}{(\eta-\xi)}\{\ln \eta+\Theta-\ln (1+\xi)\}=\frac{1}{\eta}-\frac{(1-\gamma) \Theta}{\eta}
$$

We also have continuity in $V_{x x}$ at $x=\eta y$ and so $-1+\Theta(1-\gamma)(2-\gamma)=0$. Combining these expressions, and now writing $\eta^{*}, \xi^{*}, \Theta^{*}$ for the values fixed by the smooth pasting conditions we have

$$
\Theta^{*}=\frac{1}{(1-\gamma)(2-\gamma)}
$$

as in (12). Also $\frac{\eta^{*}}{1+\xi^{*}}=1-\frac{1}{2-\gamma}=\frac{1-\gamma}{2-\gamma}$ so that

$$
\xi^{*}=\frac{\eta(2-\gamma)}{(1-\gamma)}-1
$$

Then

$$
\ln \frac{\eta^{*}}{\left(1+\xi^{*}\right)}+\Theta^{*}=\frac{\left(\eta^{*}-\xi^{*}\right)}{\left(1+\xi^{*}\right)}=-\frac{1}{2-\gamma}+\frac{(1-\gamma)}{(2-\gamma)} \frac{1}{\eta^{*}}
$$

which upon substitution for $\Theta^{*}$ gives (10), and (11) follows. 